\title{
Development and future directions of antiangiogenic therapy in hepatocellular carcinoma
}

\author{
Akinobu Taketomi ${ }^{1}$ \\ Received: 28 January 2016 / Accepted: 14 February 2016 / Published online: 20 February 2016 \\ (C) Japan Society of Clinical Oncology 2016
}

\section{Introduction}

Angiogenesis is a promising therapeutic target in treatments aimed at inhibiting tumor growth. Since the study published by Folkman in 1971 that demonstrated that tumor growth is angiogenesis-dependent [1], research has focused on identifying new therapeutic agents that can inhibit angiogenesis. In 2004, bevacizumab was developed as a potent inhibitor of vascular endothelial growth factor (VEGF), and this agent demonstrated modest antitumor activity when combined with chemotherapy. Currently, inhibition of angiogenesis has become a standard treatment option for several tumor types, including colorectal cancer, glioblastoma and ovarian cancer.

Hepatocellular carcinoma (HCC), in which hypervascularization is a major characteristic, has been a candidate for antiangiogenetic therapy. Sorafenib is an anticancer agent that suppresses angiogenesis by inhibiting the activities of RAF kinase and VEGF receptors. Two placebo-controlled, Phase III studies (SHARP and Asia-Pacific) were milestones in the treatment of patients with advanced $\mathrm{HCC}$, revealing that sorafenib caused significant improvements in overall survival [2, 3]. Based on the promising results of these clinical trials, sorafenib has been established as a first-line therapy in patients with advanced HCC. However, the subsequent phase III trial of antiangiogenic agents in HCC has failed to show improved survival compared with the standard treatment. Future research is expected in optimizing the antiangiogenic therapy for HCC to improve survival.

Akinobu Taketomi

taketomi@med.hokudai.ac.jp

1 Department of Gastroenterological Surgery I, Graduate School of Medicine, Hokkaido University, North 15 West 7, Kita-Ku, Sapporo 060-8638, Japan
Although the proliferation of tumor cells has been studied extensively, little is known about the proliferation of tumor endothelial cells (TEC) and their contribution to tumor angiogenesis [4]. Identifying molecules that are specific for TEC is important for the development of antiangiogenic therapies and would improve their efficacy.

In this issue of The International Journal of Clinical Oncology, the first article is a review of the field of TEC. The authors focused on the heterogeneity of TEC according to the tumor status in addition to the differences compared with normal endothelial cells. The second article is a review of the antiangiogenic therapies for $\mathrm{HCC}$ with a special reference to clinical trials. We hope that these special combined reviews will provide valuable information in the field of antiangiogenic treatment and will help readers to understand the current advances and future prospectives in the treatment of HCC.

\section{Compliance with ethical standards}

Conflict of interest The author declares that he has no conflict of interest.

\section{References}

1. Folkman J (1971) Tumor angiogenesis: therapeutic implications. N Engl J Med 285:1182-1186

2. Llovet JM, Ricci S, Mazzaferro V et al (2008) Sorafenib in advanced hepatocellular carcinoma. N Engl J Med 359:378-390

3. Cheng AL, Kang YK, Chen Z et al (2009) Efficacy and safety of sorafenib in patients in the Asia-Pacific region with advanced hepatocellular carcinoma: a phase III randomised, double-blind, placebo-controlled trial. Lancet Oncol 10:25-34

4. Yamada K, Maishi N, Akiyama K et al (2015) CXCL12-CXCR7 axis is important for tumor endothelial cell angiogenic property. Int J Cancer 137:2825-2836 